\title{
Efecto del SITP sobre las estructuras de pavimento flexible en la ciudad de Bogotá: un caso de estudio
}

\section{Case study: Effect of SITP in flexible pavement structures in Bogotá}

\section{Hugo Alexander Rondón Quintana}

Ingeniero Civil, doctor en Ingeniería. Docente de la Universidad Distrital Fran-

cisco José de Caldas. Bogotá, Colombia. Contacto:harondonq@udistrital.edu.co

\section{Carlos Felipe Urazán Bonells}

Ingeniero Civil, doctor en Gestión Territorial e Infraestructura de Transporte. Docente de la Universidad de La Salle. Bogotá, Colombia.

Contacto:caurazan@unisalle.edu.co

\section{Edgar Humberto Sánchez Cotte}

Ingeniero Civil, magíster en Ingeniería Civil. Docente de la Universidad Distrital Francisco José de Caldas. Bogotá, Colombia.

Contacto:ehsanchezc@udistrital.edu.co

Fecha de recepción: 30 de enero de 2013

Clasificación del artículo: caso de estudio

Palabras clave: sistema integrado de transporte público, pavimentos flexibles, movilidad, transporte.

Key words: integrated public transport system, flexible pavements, mobility, transportation.

\section{RESUMEN}

La puesta en marcha del Sistema Integrado de Transporte Público (SITP) en Bogotá D.C., requerirá revisar los actuales procedimientos de diseño y mantenimiento de las estructuras de pavimento en los principales corredores por los que transitarán las rutas de transporte público. Se espera que con el SITP los conductores de los buses adopten una postura más apropiada en el tránsito: uso más intensivo del primer carril -costado derecho- y el uso de paraderos obligatorios. Estas tendencias junto con la novedad de una menor flota vehicular y mayor ocupación generarán efectos negativos sobre las estructuras de pavimento, reflejando la necesidad de incrementar las dimensiones o espesores de dichas estructuras, realizar mantenimiento más frecuente o rehabi- 
litación anticipada. Los aspectos reportados en el presente estudio servirán para que los organismos distritales correspondientes los tengan presente en el diseño, construcción y mantenimiento de las vías bogotanas.

\section{ABSTRACT}

The implementation of Integrated Public Transport System (SITP) in Bogotá D.C. requires reviewing the pavement design and maintenance in the main corridors through which circulate public transport lines. It is expected that SITP bring an appropriate transit behavior to the buses drivers: The use of the right side lane and the mandatory bus stops. These trends along with the lower fleet and higher occupancy generate negative effects on pavement structures, showing the need to increase the dimensions or thickness of the pavements. The aspects reported in this study will assist to district agencies to include the roads design, construction and maintenance in Bogotá D.C.

\section{INTRODUCCIÓN}

En el 2012 comenzó a regir el Sistema Integrado de Transporte Público (SITP) en Bogotá, de acuerdo con la Secretaría Distrital de Movilidad (SDM), algunos de los beneficios de la introducción del SITP para Bogotá [1] son: 1) la reducción y modernización del parque automotor, se reducirá aproximadamente, en respuesta a la sobre oferta actual, en un $23 \%$ : 12333 buses, 3667 menos que los que ruedan actualmente (16000 buses) [2], en el presente, la ocupación promedio en hora pico de los buses es de $68 \%$. Con respecto a la modernización de vehículos de transporte se resalta que, en el 2007, la edad promedio de flota era de 11,4 años y se quiere pasar con el SITP a un promedio de 5 años; 2) fin de la "Guerra del Centavo"; 3) ampliación de cobertura especialmente en zonas periféricas de la ciudad; 4) mejora en la calidad del servicio: responsabilidad y control de niveles de servicio; 5) reducción de la contaminación debido a la disminución del parque automotor de transporte y a su modernización; 6) generación de empleo formal y 7) mejora la calidad de vida en la ciudad con la mejora en la movilidad producto de la reducción de los buses utilizados en el actual sistema de transporte urbano.
El presente artículo no tiene como fin criticar el SITP, ya que sus autores lo consideran como un sistema necesario para mejorar la organización del transporte y la movilidad de la ciudad. Por este motivo, el objetivo de este trabajo es presentar algunos aspectos que se deben tener en cuenta para no poner en riesgo la infraestructura vial de la ciudad, especialmente en lo que concierne al incremento en las cargas que generará el sistema sobre las estructuras de pavimentos. En especial, se dará respuesta en este artículo a las siguientes inquietudes que surgen en los autores con la implementación del sistema y su operación en las vías principales de la ciudad: a) la disminución de la oferta es equivalente a buses del SITP más cargados, es decir, el sistema presentará menos vehículos operando pero más cargados, ¿lo anterior incide en el deterioro de los pavimentos?; b) si la ocupación promedio de los buses que integrarán el SITP es del $100 \%$, ¿cómo incide esto en el daño que experimentarán las estructuras del pavimento? Esta afirmación se ampara en la reducción de la flota vehicular que pasará a un $77 \%$ de la flota actual [2], pero la demanda de pasajeros se mantiene, por lo que es presumible que igual número de usuarios en menos buses implique un mayor nivel de ocupación; c) ¿qué sucede si en el SITP no se respetan los pesos máximos permitidos de carga? y d) con el SITP, los buses 
circularán por el carril derecho y a menor velocidad, ¿qué efectos trae sobre las estructuras viales este hecho? Lo anterior para tener en cuenta que en el sistema actual no todos los buses circulan por el carril derecho y debido a la "Guerra del Centavo" en muchas ocasiones las velocidades de circulación son altas (superiores a $60 \mathrm{~km} / \mathrm{h}$ ).

Para dar respuesta a las anteriores inquietudes, se realizarán simulaciones del daño que experimentarán las estructuras de pavimento flexible de la ciudad de Bogotá, con la implementación del SITP. Este daño será deducido en términos del cálculo de la variable tránsito, la cual es una de las principales variables del diseño de estructuras de pavimentos. Inicialmente, el artículo introduce al lector en aspectos concernientes al SITP y presenta de manera resumida la forma como se calcula la variable tránsito para luego presentar las simulaciones que se realizaron con el fin estimar los efectos que generarán las nuevas cargas del sistema SITP sobre las estructuras de pavimentos flexibles de las vías principales de la ciudad.

Los estudios consultados sobre el SITP se centran principalmente en aspectos operativos y socioeconómicos [3] - [5], así como en el impacto positivo sobre el medio ambiente [6]. No se encontraron estudios similares al presente que analicen el impacto que tiene la reorganización del sistema de transporte público sobre la afectación en las estructuras de pavimentos. Lo que se reportan son estudios que muestran como las cargas, presiones de contacto y la forma como se distribuyen dichas cargas influyen en el estado de esfuerzos y deformaciones que experimentarán las estructuras viales [7] - [14].

El presente estudio cobra importancia toda vez que, de acuerdo con el Instituto de Desarrollo Urbano, IDU [15], la malla vial a diciembre de 2009, en la ciudad de Bogotá D.C., es de 15657,3 $\mathrm{km}$-carril, de los cuales, el 94,53\% se encuentra en mal estado y el 63,2\% en estado regular (malo
$40,3 \%$ y regular $22,9 \%$ ). La idea es entonces presentar simulaciones que pongan en evidencia los efectos que puede traer la implementación del SITP sobre los pavimentos, de tal forma que se ejecuten las actividades necesarias por parte de las entidades del Estado para no incrementar el habitual deterioro de la actual malla vial de la ciudad. Adicionalmente, en Bogotá D.C., en los últimos cinco años el número total de vías nuevas solo se ha incrementado en un $2 \%$, el de vehículos particulares lo ha hecho en un $58 \%$. De esta manera, el índice de ocupación en las vías se habría incrementado en más de un $25 \%$ en este período. El tema es aún más crítico si se tiene en cuenta que los recursos presupuestales no resultan suficientes para mantener en buen estado las vías existentes [16]. En el mismo [16] se menciona que, según el Plan Maestro de Movilidad del Distrito, el sistema de transporte de la Región Capital tiene como eje estructural al Sistema Integrado de Transporte Público (SITP), armonizado y soportado en estrategias que favorezcan objetivos, entre los que se cuenta la necesidad de terminar con la sobreoferta de transporte público con el consiguiente impacto sobre la reducción de la congestión, contaminación, accidentalidad, deterioro del pavimento y la mejora en la sostenibilidad financiera de la actividad.

Es importante resaltar que las condiciones de transporte público e infraestructura vial referentes a la ciudad de Bogotá D.C., pueden corresponder a escenarios de otras grandes ciudades en el mundo. Por lo tanto, el planteamiento y los resultados obtenidos en el presente estudio pueden servir como referente para la planificación del desarrollo de otras urbes.

\section{GENERALIDADES DEL SITP}

En el caso de la ciudad de Bogotá D.C., el SITP tiene su sustento en el Plan Maestro de Movilidad y en el decreto 319 de 2006 [17]. Ese decreto 
otorga la responsabilidad de gestor del SITP a la empresa de Transporte del Tercer Milenio-TransMilenio (TM). Posteriormente, el decreto 309 de 2009 -por el cual se adopta el SITP, [18]-, considera la reorganización del transporte colectivo en la ciudad a través de la entrega en concesión del servicio en el escenario de trece zonas en la ciudad: Usaquén, Suba Oriental, Suba Pueblo, Calle 80, Engativá, Fontibón, Kennedy Norte, Kennedy, Bosa, Perdomo, Ciudad Bolívar, Usme y San Cristóbal [18].

Bajo este nuevo esquema operacional, se considera una remuneración al transportador distinta al pago por usuario transportado, lo que implica mejores índices de calidad del servicio por puntualidad y seguridad vial al dar fin a la denominada "Guerra del Centavo" que se traduce en una competencia entre conductores por captar el mayor número de pasajeros posibles.

Como un segundo beneficio, el SITP proyecta una tarifa integrada que permite al ciudadano el uso de cualquier modo de transporte público colectivo y masivo en la ciudad, con la posibilidad de un costo adicional por transbordo realizado. En este esquema, el dinero efectivo deja de ser válido y se considera un recaudo centralizado que se soporta a través de una tarjeta única de acceso con servicio prepago.

Otro cambio que trae consigo el SITP es el uso de paraderos obligatorios, a diferencia del escenario actual en el que, en la mayoría de rutas de transporte colectivo, el bus realiza paradas para ascenso y descenso de pasajeros en el lugar que el usuario indique con una seña de mano [19].

\subsection{Esquema financiero y de transición}

El SITP se proyecta como un sistema que debe ser financieramente sostenible -no debe disponer de recursos públicos para la operación-. Sin embargo, se ha considerado un "subsidio" del estado para infraestructura vial, seguridad, aseo y regulación del precio de combustibles (Fondo de Compensación). El sistema también debe garantizar cobertura total, funcionalidad las 24 horas al día y garantizar la capacidad de transporte de pasajeros en horas pico a través de rutas troncales y alimentadoras (TM), pretroncal, auxiliares, complementarias y especiales -nuevas rutas-, bajo la gestión de TM.

A partir de la puesta en marcha del SITP, se iniciará un proceso de transición de un (1) año, que le permitirá a los nuevos operarios de las rutas, evaluar las condiciones del nuevo esquema operacional, ajustar sus modelos económicos, legalizar la inclusión de los propietarios a las sociedades, trámite de créditos, chatarrización de buses y compra de nuevas unidades de transporte, definición estructura organizacional y operativa, evaluación de rutas y valoración de la demanda. Terminado este proceso, se deja en firme el contrato de concesión y se inicia el proceso de operación por un tiempo de 24 años.

Se estima que la flota inicial contará con 1959 microbuses, 1520 busetas, 4550 buses, 2729 buses padrones, 1306 articulados y 207 biarticulados. Por tanto, quedarán operando en el SITP más de 12200 unidades vehiculares, de las cuales 8000 circulan actualmente -menos de 10 años- y serán adecuados al sistema de pago, y los otros 4.000 serán vehículos nuevos [2].

\subsection{Tecnología vehicular}

En cumplimiento de la regulación nacional, las nuevas unidades de transporte deberán responder a especificaciones Euro IV o superior. Esta tecnología sumada a una operación regulada de la flota, control de la edad (diez años) y mantenimiento para control emisiones, permitirá una reducción de emisiones contaminantes, esto sin considerar 

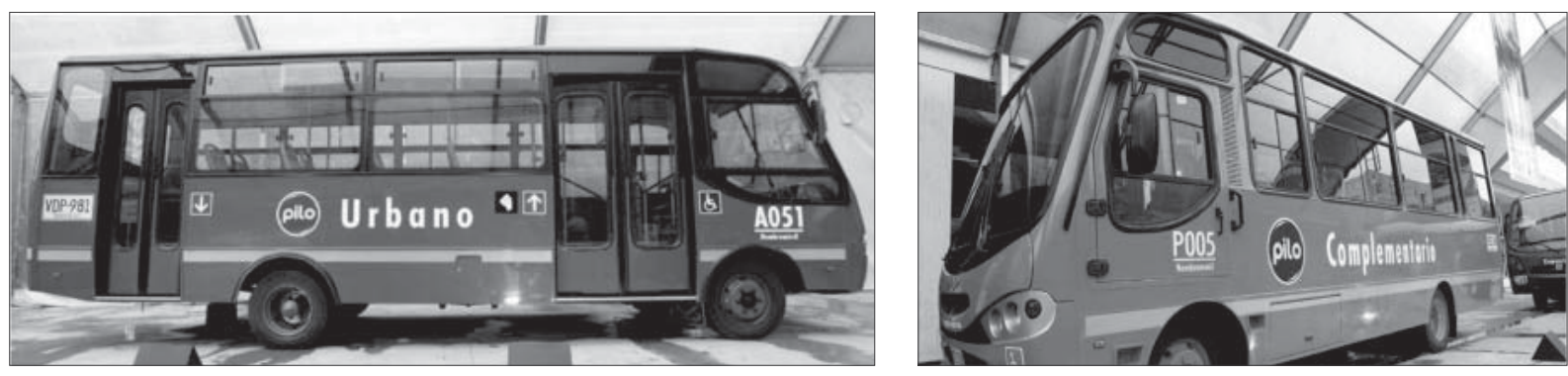

Figura 1. Algunos de los buses que operarán con el SITP

Fuente: El Tiempo, 16 de noviembre de 2011

los beneficios de incluir en la operación unidades de transporte eléctricas. Además, se considera un programa de contratación de personal ajustado a la legislación en cuanto a horas de trabajo, pago de salud y prestaciones laborales que dignifica la labor del conductor.

\subsection{Sistema de pago integrado}

El SITP también contará con un Sistema Integrado de Recaudo, Control e Información y Servicio al Usuario (SIRCI) que tendrá como responsabilidad el manejo de la tarjeta integrada de acceso, el recaudo de dineros y el sistema de información para el usuario. Actualmente se estima que los dineros a recaudar por el SIRCI corresponden a 2,8 billones de pesos anuales [20].

\subsection{Tipos de buses y servicio}

Los vehículos con los que operará el SITP se pueden describir en 5 tipologías: biarticulado ( 240 pasajeros), articulado (160 pasajeros), padrón (90 pasajeros), bus (50 pasajeros), buseta (40 pasajeros), microbus (19 pasajeros). Los buses también se pueden clasificar por color de carrocería, en función del tipo de la extensión y localización de las rutas: azul para las vías principales, naranja para rutas entre localidades, y vinotinto que irán por zonas donde hoy no se presta el servicio (figura 1).
Los buses prestarán cuatro tipos de servicio:

a. Troncal y pretroncal: atenderá los corredores de mayor demanda y de mayor distancia, con servicio en estaciones. Este servicio lo prestarán buses articulados y biarticulados.

b. Auxiliar: complementa la oferta de las rutas troncales y pretroncales en corredores de demandas medias. Este servicio lo prestarán buses tipo padrón.

c. Alimentador y complementario: apoya a las rutas troncales, conectando a los portales y estaciones intermedias con zonas cercanas. Este servicio lo prestarán buses tipo bus, buseta y microbús.

d. Especial: se prestará con buses tipo microbús y tiene como finalidad cubrir sectores de difícil acceso, como el caso de los cerros y zonas de baja demanda.

Solo los servicios troncales y pretroncales contarán con servicio en estaciones cerradas y en portales, con pago del pasaje validando en la entrada a la estación — actual esquema de TM-.

Por su parte, los servicios alimentador, complementario, auxiliar y especial, serán atendidos en paraderos abiertos, y el pago del pasaje se efectuará al interior del bus, previo pago para pasar por el torniquete de acceso (figura 2). 


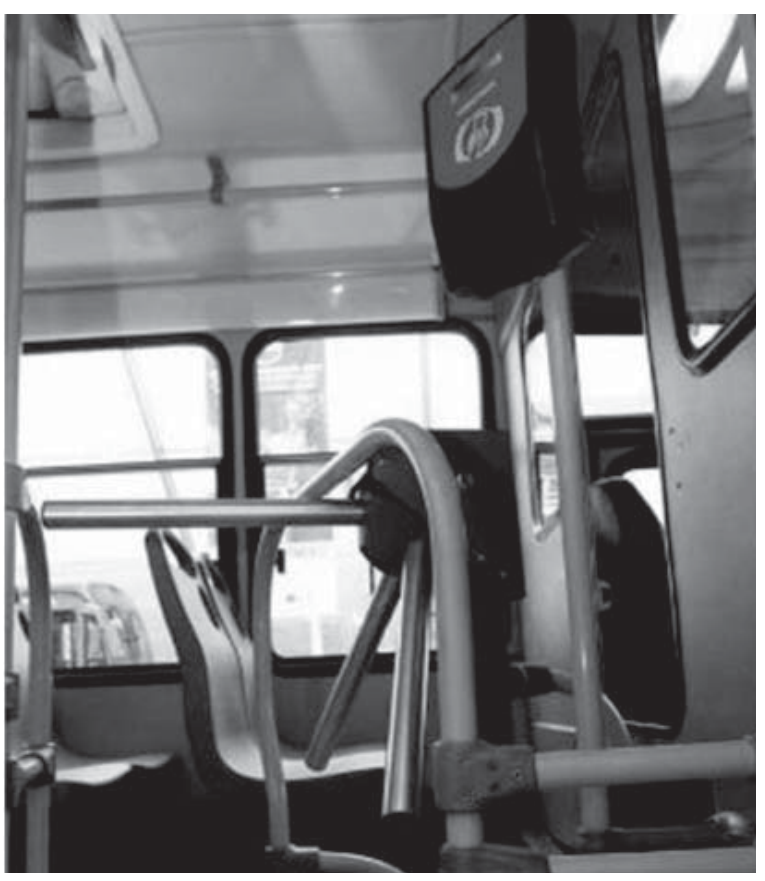

Figura 2. Torniquete de acceso y dispositivo lector de tarjeta de pago en los buses del SITP

Fuente: El Tiempo, 16 de noviembre de 2011

\section{VARIABLE TRÁNSITO}

La variable tránsito $(N)$ se calcula realizando el conteo y pesaje de vehículos y ejes de carga que circulan por una vía durante un periodo determinado de tiempo -generalmente una semana o un día-, para luego ser proyectados al periodo de diseño con el fin de estimar el número de ejes de carga que circulará por la vía durante la vida útil de la estructura de pavimento. Adicionalmente, estos ejes se convierten al daño que generan con respecto a un eje de referencia. Para el cálculo de esta variable se utiliza por lo general la ecuación (1).

$$
N=T P D \times \frac{k_{1}}{100} \times \frac{k_{2}}{100} \times 365 \times \frac{(1+r)^{n}-1}{\ln (1+r)} \times F C
$$

\section{Donde}

$N$ es el número de ejes equivalentes de 8,2 toneladas que circulan por el carril y el periodo de diseño
TPD o Tránsito Promedio Diario es el número de vehículos que circulan por una vía en un día $k_{1}$ es el porcentaje de vehículos pesados (buses y camiones)

$k_{2}$ es el factor carril y tiene en cuenta el porcentaje de vehículos que circularán por el carril de diseño de la vía -se escoge el carril derecho ya que por allí es por donde se movilizan en teoría los vehículos pesados y circulan a menor velocidad generando cargas más agresivas sobre el pavimento- $r$ es la tasa de crecimiento anual del tránsito $n$ es el periodo de diseño en años y

$F C$ es el factor camión y tiene como función, inicialmente, pasar el número de vehículo a número de ejes con su respectiva masa para luego convertir el daño que genera cada eje en comparación con el de referencia (8,2 toneladas).

Cada eje de carga que circula por una vía presenta pesos, configuraciones y presiones de inflado de llanta diferentes, los cuales aportan a la estructura un grado de agresividad y deterioro distinto -medido principalmente en términos de fatiga y ahuellamiento para el caso de pavimentos flexibles-. Por lo general, para el cálculo de la variable tránsito, este grado de agresividad de cada eje de carga se convierte al que, en teoría, producirá un eje estándar equivalente de 8,2 t. -este eje es derivado de los resultados de los tramos de prueba construidos y monitoreados por la AASHO Road Trials [21] en el estado de Illinois, Estados Unidos, y sobre los cuales se fundamenta la metodología de la AASHTO [22]-. Esta conversión se realiza de manera simplista, multiplicando el número de repeticiones de carga de cada eje por un factor de equivalencia $(f)$ o factor de daño. Colombia utiliza como factor la ecuación (2) para el caso de ejes simples de cuatro ruedas -dos en cada esquina del eje- y estructuras de pavimentos flexibles, y es derivado de los resultados reportados por la AASHO Road Trials [21]. 


$$
f=\left(\frac{p}{8,2}\right)^{4}
$$

Donde $p$ es la masa del eje en toneladas, cuyo grado de agresividad será convertido al de 8,2 toneladas.

Para el caso de ejes simples de dos ruedas -una encada esquina del eje- el denominador de la ecuación (2) se cambia por 6,5 .

En la figura 3 se presenta la representación gráfica de la ecuación (2). Se observa que la agresividad que genera el parque automotor sobre las estructuras de pavimentos incrementa de manera quasi-exponencial cuando se aumenta la carga. Analizando las ecuaciónes (1) y (2), se evidencia de manera obvia que la variable tránsito crece de manera lineal con el número de vehículos pesados que circulen por una vía, mientras que éste crecimiento es quasi-exponencial con respecto a la carga. De lo anterior, se deduce que el deterioro de los pavimentos se ve afectado más por el incremento de cargas sobre estas estructuras viales que por el número de vehículos que circulen sobre ellas. Para entender con mayor claridad lo anteriormente mencionado, a continuación se describen los siguientes tres casos que fueron obtenidos realizando simulaciones en donde se emplean las ecuaciónes (1) y (2). Estos casos fueron preparados teniendo en cuenta que, en Colombia, el peso máximo permitido de carga de buses urbanos y metropolitanos -se exceptúan los buses articulados- que pueden circular por una estructura vial es de 17 toneladas, las cuales se distribuyen de la siguiente forma: 6 toneladas en el eje simple delantero y 11 toneladas en el eje simple trasero -se supone en la simulación que ambos ejes presentan una rueda en cada esquina-.

- Caso 1. El daño que produce cada eje de un bus que circule con el peso máximo permitido equivale a 4,68 veces el daño que producen

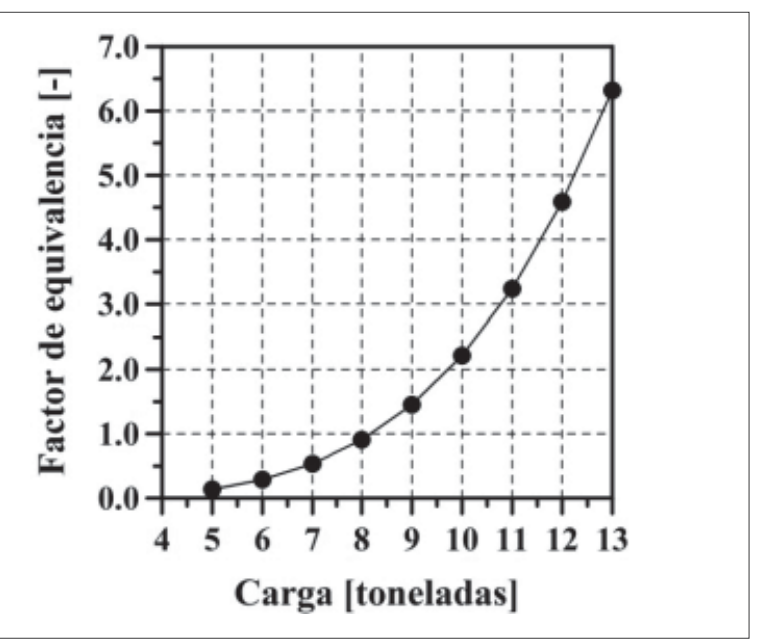

Figura 3. Evolución del factor de equivalencia f con la carga en toneladas para ejes simples de dos ruedas

Fuente: elaboración propia.

los mismos ejes del mismo bus pero cargado al $68 \%$ de su carga máxima permitida.

- Caso 2. El daño que produce cada eje de un bus que circule con el peso máximo permitido equivale a 123,5 veces el daño que producen los mismos ejes del mismo bus pero cargado al $30 \%$ de su carga máxima permitida.

- Caso 3. Los ejes de un bus cargado al máximo de su capacidad genera entre 625 y 10000 veces el daño que genera el mismo bus pero con el $20 \%$ y el $10 \%$ de su peso respectivamente.

\section{SIMULACIONES}

En el sistema de transporte actual, por las vías principales, los vehículos de transporte se distribuyen aproximadamente de la siguiente forma: $25 \%$ de busetas y $75 \%$ de buses -se excluyen los microbuses por ser una cantidad insignificanteComo ya se mencionó, la reducción del total de vehículos de transporte con la implementación del SITP será del $23 \%$ aproximadamente. El $77 \%$ 
restante que circulará por las vías de Bogotá D.C., se distribuirán de la siguiente manera: 15,96\%, $12,39 \%, 37,08 \%, 22,24 \%, 10,64$ y $1,69 \%$ para microbuses, busetas, buses, padrones, articulados y biarticulados respectivamente. Para el caso de las vías principales, entonces, del $77 \%$, circularán el $71,71 \%$ de los vehículos (busetas, buses y padrones). De este $71,71 \%$, las busetas, los buses y los padrones representarán el 17,27\%, 51,71\% y $31,01 \%$ respectivamente.

De acuerdo con Transmilenio [23], los buses padrones tienen como capacidad máxima de carga en el eje delantero y trasero 7,5 y 12,5 toneladas respectivamente, obteniendo un peso bruto vehicular máximo de 20 toneladas. Algunas otras características de dichos buses son las siguientes:

- Capacidad mínima total (sentados y de pie) de ochenta (80) pasajeros.

- Ejes delantero y trasero compuestos por dos y cuatro llantas respectivamente.

- Número total de asientos: mínimo $40 \%$ de la capacidad total del vehículo.

- Área mínima interna libre pasajeros de pie: $9 \mathrm{~m}^{2}$.

- 7 Pasajeros de pie por $\mathrm{m}^{2}$.

- Para el cálculo de pesos de los buses padrón, se tiene en cuenta una masa por pasajero de $68 \mathrm{~kg}$.

En el caso de los buses y busetas los pesos se mantienen con respecto al sistema actual:

- Para los buses la capacidad máxima de carga en el eje delantero y trasero son de 6 y 11 toneladas respectivamente, obteniendo un peso bruto vehicular máximo de 17 toneladas. Ejes delantero y trasero compuestos por dos y cuatro llantas respectivamente.
- Para las busetas, la capacidad máxima de carga en el eje delantero y trasero son de $5 \mathrm{y}$ 11 toneladas respectivamente, obteniendo un peso bruto vehicular máximo de 16 toneladas. Ejes delantero y trasero compuestos por dos y cuatro llantas respectivamente.

Con base en la información presentada con anterioridad y teniendo en cuenta las ecuaciones (1) y (2) se hace evidente lo siguiente:

- Simulación 1. En horas punta, de acuerdo con la Secretaría Distrital de Movilidad (SDM) [1], en el sistema SITP la tendencia de los buses será ir al $100 \%$ de su capacidad mientras que en la actualidad en este espacio de tiempo solo se llega al $68 \%$. Eso significa que en las horas pico la variable tránsito ( $N$ en la ecuación (1)) incrementará 5,3 veces aproximadamente.

- Simulación 2. Actualmente, en horas valle, la tendencia de los buses es circular con el 35\% de su capacidad aproximadamente. Suponiendo que en el sistema SITP la tendencia de los buses será ir al $80 \%$ de su capacidad, la variable tránsito incrementaría entonces en 31 veces aproximadamente.

En las dos simulaciones presentadas se tuvo en cuenta adicionalmente que, en el sistema SITP, todos los buses circularán por el carril derecho mientras que en la actualidad los buses circulan en todos los carriles que componen la calzada de la vía. Esto genera que la agresividad o el daño sobre este carril, que es el de diseño, incremente. Normalmente se considera, para el caso de una vía de seis carriles -tres en cada dirección-, que del total de los buses que circulan por la vía, el $80 \%$ aproximadamente transitan por el carril derecho, sin embargo, con el SITP este porcentaje se aumenta a $100 \%$.

- Simulación 3. Suponiendo un TPD de 5000 buses diarios -se eliminan los automóviles y 
camiones para simplificar la simulación- que circulen por una vía de seis carriles (tres en cada dirección), $r=3 \%, n=10$ años, para las condiciones de subrasante $(C B R \cong 3 \%)$ y clima de Bogotá D.C. (temperatura media anual promedio, $T M A P=13^{\circ} \mathrm{C}$ ), se obtiene que en el sistema SITP, la estructura de pavimento debe aumentar en $10 \mathrm{~cm}$ las capa asfáltica y $5 \mathrm{~cm}$ las capa de base granular aproximadamente con respecto a la estructura que debe soportar el sistema actual.

La pregunta que surge como respuesta a las simulaciones presentadas con anterioridad es la siguiente: ¿El SITP está teniendo en cuenta el daño que puede producir el nuevo sistema de transporte sobre la infraestructura vial de la ciudad?

Si en un futuro, los buses y busetas que transitan actualmente por las vías principales de la ciudad quieren ser reemplazados por buses padrones, se debe tener en cuenta lo siguiente:

- Simulación 4. La variable tránsito $N$ que producen los ejes delantero y trasero de un bus padrón que circule con el peso máximo, equivale a 2,44 y 1,67 veces aquella que producen los mismos ejes de un bus tipo que circule actualmente por la ciudad con su peso máximo permitido de carga. El $N$ es de 5,06 y 1,67 veces cuando se comparan con los ejes de una buseta.

- Simulación 5. En horas punta, un bus padrón deberá ser sustituido por al menos 10 buses o 16 busetas de las actuales para generar el mismo daño sobre el pavimento.

- Simulación 6. En horas valle un bus padrón deberá ser sustituido por al menos 56 buses o 92 busetas de las actuales para generar el mismo daño sobre el pavimento.

- Simulación 7. Si las cargas máximas de los buses del SITP no se controlan y se incre- mentan en un $2,5 \%$ y $5 \%$, éste incremento genera un aumento del $10,38 \%$ y $21,55 \%$ en la variable tránsito respectivamente.

El efecto del mayor deterioro de las estructuras viales producto de la disminución de la velocidad de los nuevos buses del sistema SITP es complejo de simular y por lo tanto no se presenta en el documento. Sin embargo, es importante que, a futuro, las entidades del Estado tengan en cuenta dicho efecto en el dimensionamiento de las estructuras de pavimentos flexibles por las cuales circularán los buses del SITP. Un estudio realizado para evaluar dicho efecto sobre un tipo de mezcla asfáltica colombiana puede ser consultado en Rondón [24].

\section{CONCLUSIONES}

La flota vehicular dispuesta para el SITP en Bogotá D.C. operará para atender una demanda de pasajeros que se puede considerar igual o similar a la que se cuantifica actualmente. Ese escenario se traduce en menos buses, pero más cargados. Esa mayor carga u ocupación vehicular es estimada por fuentes oficiales del Distrito en un incremento del $68 \%$ al $100 \%$ en hora punta, implicando que cada vehículo tipo bus -para ejemplificar un caso- produce un daño a la estructura del pavimento, correspondiente a un incremento de 5,3 veces en la variable tránsito. No obstante, las consecuencias podrían ser peores, curiosamente, en horas valle. Si la actual ocupación de los buses en hora valle (35\%) se incrementa al $80 \%$ en la misma franja horaria, la variable tránsito incrementará en 31 veces.

Por otro lado, el SITP estima un aumento de la flota de buses tipo padrón, los cuales, con ocupación del $100 \%$, generarían variables tránsito en la estructura del pavimento que puede llegar a ser 2,44 veces superior a la que produce un 
vehículo tipo bus, y 5,06 veces superior al de un vehículo tipo buseta.

Si las cargas máximas de los buses que circularán en el SITP no se controlan, y se incrementan en un $2,5 \%$ y $5 \%$, éstos incrementos generarán respectivamente un aumento aproximado del 10,38\% y $21,55 \%$ en la variable tránsito.

Los cambios que se asume que trae el SITP para las cargas impuestas al pavimento, expuestos en un escenario como el de la carrera séptima de Bogotá D.C., con condiciones de temperatura y estructuras flexibles estándar, muestran la necesidad de que el diseño incremente en $10 \mathrm{~cm}$ la capa asfáltica y en $5 \mathrm{~cm}$ la capa de base granular.

Adicionalmente se deduce que la cantidad de unidades vehiculares que saldrán de circulación con el SITP no serán suficientes para equivaler el daño que produce la flota actual en las principales vías no troncales de Bogotá.
Los anteriores argumentos dejan entrever la urgente necesidad de que las autoridades distritales vayan preparando un plan de mantenimiento y reforzamiento estructural para las vías de mayor afectación por la red del SITP, que sea adecuado a las nuevas condiciones de carga de los buses. Para ello, se ha de cuantificar la flota vehicular a medida que se vaya incorporando al sistema, realizar aforos frecuentes del nivel de ocupación vehicular, así como registros de velocidades de marcha y ocupación de carriles por parte de la nueva flota. Estos registros serán una herramienta fundamental para poder precisar los efectos de daño sobre la malla vial afectada y su correspondiente plan de acción y mantenimiento.

En la bibliografía consultada, no se encontraron estudios similares al presente que analicen el impacto que tiene la reorganización del sistema de transporte público sobre la afectación en las estructuras de pavimentos.

\section{REFERENCIAS}

[1] SDM - Secretaría Distrital de Movilidad, El SITP: Bogotá se Mueve Bien, [Taller - Conversatorio sobre el Esquema Empresarial para la Operación del Sistema Integrado de Transporte Público - SITP], Cámara de Comercio: Bogotá D.C., 26 de septiembre de 2007.

[2] Diario EL TIEMPO, Presentaron los buses que rodarán con el sistema integrado, edición del 16 de noviembre de 2011, [en línea]. Disponible en: http:// www.eltiempo.com/colombia/bogota/ ARTICULO-WEB-NEW_NOTA_INTERIOR-10777145.html.

[3] J. Acevedo, "Resumen del libro: El transporte como soporte al desarrollo de Co- lombia. Una Visión al 2040", Revista de Ingeniería, No.29, pp. 156-162, 2009.

[4] J. Lupano y R. Sánchez, Políticas de movilidad urbana e infraestructura básica de transporte, CEPAL (ONU), 2009.

[5] G. Correa,"Transporte y ciudad", Revista EURE, Santiago de Chile, Vol. 36, No. $107,2010$.

[6] F. Franco, "Contaminación atmosférica en centros urbanos. Desafíos para lograr su sostenibilidad: caso de estudio Bogotá", Revista EAN, No. 72, pp. 193-204, 2012.

[7] S. Price, Y. Mehta and L. M. McCarthy, "Evaluation and modeling of repea- 
ted load test data of asphalt concrete for mechanistic-empirical pavement design", Journal of Materials in Civil Engineering, Vol. 19, No. 11, pp. 993-999, 2007.

[8] A. Papagiannakis and E. Masad, Pavement design and materials, Ed. John Wiley \& Sons Inc., 542 p., 2007.

[9] S. Seeds, Flexible pavement design: summary of the state of the Art, Transportation Research Board, A3B05: Committee on Safety Data, Analysis, and Evaluation, 2000 .

[10] U. Seyhan, E. Tutumluer and H. Yesilyurt, "Anisotropic aggregate base inputs for mechanistic pavement analysis considering effects of moving wheel loads", Journal of Materials in Civil Engineering, Vol. 15, No. 5, pp. 505-512, 2005.

[11] P. Ullidtz, Pavement analysis. Development in civil engineering, 19. Ed. Elsevier, 318 pp., 1987.

[12] I. Vera, G. Thenoux, H. D. Solminiach y T. Echaveguren, "Modelo de evaluación técnica del desempeño del mantenimiento de pavimentos flexibles", Revista de la Construcción, Santiago de Chile, Vol. 9, No. 2, pp. 78-88, 2010.

[13] E. J. Yoder, M. W. Witczak, Principle of pavement design, Wiley, New York, 1975.

[14] Y. H. Huang, Pavement analysis and design, second edition, Pearson Prentice Hall, Upper Saddle River, NJ., 2004.

[15] Instituto de Desarrollo Urbano (IDU), Bogotá, 2009, [en línea]. Disponible en: http://www.idu.gov.co/web/guest/malla inventario
[16] CONPES 3677, República de Colombia, Departamento Nacional de Planeación, Bogotá D.C., 2010.

[17] Alcaldía Mayor de Bogotá D.C., Decreto 319 de 2006, por el cual se adopta el plan maestro de movilidad para Bogotá Distrito Capital, [en línea]. Disponible en: http://www.alcaldiabogota.gov.co/sisjur/ normas/Norma1.jsp?i=21066.

[18] Alcaldía Mayor De Bogotá D.C., 2009, Sistema Integrado de Transporte Público SITP, [en línea]. Disponible en: www.andi.com.co/downloadfile. aspx?Id=844DF215-534E-4A08.

[19] C. F. Urazán y E. A. Velandia, Cambios en las paradas del transporte público colectivo en Bogotá D.C., Asociadas ala implementación del Sistema Integrado de Transporte Público SITP, en: $10^{\circ}$ Congreso Colombiano de Ingeniería de Tránsito y Transportes, Universidad Nacional de Colombia, Medellín, 5 al 7 de noviembre de 2011.

[20] SDM - Secretaría Distrital de Movilidad, Bogotá D.C., 2010, Manual de operaciones sistema integrado de transporte público, [en línea]. Disponible en: http:// cootranspensilvania.com.co/(4)\%20 $\% 20$ Anexo\%20\%202\%20\%20\%20Manual $\% 20 \mathrm{de} \% 20$ Oper.pdf

[21] AASHO - American association of state highway officials, The AASHO road test, special report 61 (7 reports, $A-G)$, National academy of sciences - National research council, Washington D.C., 1961.

[22] AASHTO - American association of state highway and transportation officials, Guide fordesign of pavement structures, Washington, D.C., 1986, 1993. 
[23] TRANSMILENIO, Alcaldía Mayor de Bogotá D.C, Manual de operaciones sistema integrado de transporte público, Bogotá D.C., 2010.
[24] H. A. Rondón, F. A. Reyes, y C. F. Urazán, "Efecto de la disminución de la velocidad vehicular en la durabilidad de una capa asfáltica", Revista Ingeniare, Vol. 23, No. 1,2013. 